\title{
Linear Parameter-Varying Model to Design Control Laws for an Artificial Pancreas
}

\author{
P. Colmegna ${ }^{\mathrm{a}, \mathrm{b}, *}$, R. S. Sánchez-Peña ${ }^{\mathrm{a}, \mathrm{c}}$, R. Gondhalekar ${ }^{\mathrm{d}}$ \\ ${ }^{a}$ CONICET, Argentina. \\ ${ }^{b}$ Departamento de Ciencia y Tecnología, Universidad Nacional de Quilmes, Buenos Aires, Argentina. \\ ${ }^{c}$ Centro de Sistemas y Control, Instituto Tecnológico de Buenos Aires, Buenos Aires, Argentina. \\ ${ }^{d}$ Harvard John A. Paulson School of Engineering \& Applied Sciences, Harvard University, Cambridge, MA, USA.
}

\begin{abstract}
The contribution of this work is the generation of a control-oriented model for insulin-glucose dynamic regulation in Type 1 Diabetes Mellitus (T1DM). The novelty of this model is that it includes the time-varying nature, and the inter-patient variability of the glucose-control problem. In addition, the model is well suited for well-known and standard controller synthesis procedures. The outcome is an average Linear Parameter-Varying (LPV) model that captures the dynamics from the insulin delivery input to the glucose concentration output constructed based on the UVA/Padova metabolic simulator. Finally, a system-oriented reinterpretation of the classical ad-hoc 1800 rule is applied to adapt the model's gain.

The effectiveness of this approach is quantified both in open- and closed-loop. The first one by computing the Root Mean Square Error (RMSE) between the glucose deviation predicted by the proposed model and the UVA/Padova one. The second measure is determined by using the $\nu$-gap as a metric to determine distance, in terms of closed-loop performance, between both models. For comparison purposes, both open- (RMSE) and closed-loop ( $\nu$-gap metric) quality indicators are also computed for other control-oriented models previously presented.

This model allows the design of LPV controllers in a straightforward way, considering its affine dependence on the time-varying parameter, which can be computed in real-time. Illustrative simulations are included. In addition, the presented modeling strategy was employed in the design of an Artificial Pancreas (AP) control law that successfully withstood rigorous testing using the UVA/Padova simulator, and that was subsequently deployed in a clinical trial campaign where five adults remained in closed-loop for 36 hours. This was the first ever fully closed-loop clinical AP trial in Argentina, and the modeling strategy presented here is considered instrumental in resulting in a very successful clinical outcome.
\end{abstract}

Keywords: LPV model, controller synthesis, type 1 diabetes, artificial pancreas, $\nu$-gap metric.

\section{Introduction}

An Artificial Pancreas (AP) is a system that automatically controls glycemia in Type 1 Diabetes Mellitus (T1DM) patients by infusing an adequate amount of insulin, according to the measured glucose level. The decision of how much insulin to infuse is made by a control algorithm. In general, this algorithm is based on a mathematical model that is required to suitably describe the insulin-glucose dynamics. Thus, the model constitutes a key element in the development of a reliable AP.

\footnotetext{
* Corresponding author.

E-mail address: patricio.colmegna@unq.edu.ar (P. Colmegna).

Preprint submitted to Elsevier
}

Several simulation models have been proposed since the late 1970s [1-4]. They have been used to perform a vast amount of in silico studies, giving an affordable and safe means of testing glucose controllers. Thus, the use of computer simulation has accelerated the development of AP [5].

The main goal of simulation models is to provide a blood glucose prediction as close as possible to a real situation. However, this class of models is not generally used for controller synthesis, due to its excessive mathematical complexity. Therefore, simplifications of these models are generally considered at the controller design phase, because most of the well established theory of control law design accommodates only simpler 
models that are normally referred to as control-oriented models. Thus, although control-oriented models have to represent the underlying dynamics to some degree, they are mainly obtained for synthesis purposes and have a much simpler mathematical formulation.

Another aspect that is worth considering in designing glucose controllers is that most metabolic parameters related to the insulin-glucose system are not easily identifiable in practice, and finding each parameter of a complex and time-varying model is intractable. Therefore, some tuning based on only a small number of easily obtainable patient-specific characteristics is required in practice for a safe and effective AP [6]. Consequently, a few works have been focused on such personalization [7-11].

One interesting approach to obtain a personalized control-oriented model is to adapt a low-order model structure based on a priori patient information. For example, given the patient's Total Daily Insulin (TDI), an insulin sensitivity factor can be obtained using the so-called 1800 rule (1800/TDI) that is suggested in the medical care literature [12]. From the medical point of view, the 1800 rule indicates the maximum drop in glucose concentration, measured in $\mathrm{mg} / \mathrm{dl}$, after a 1 $\mathrm{U}$ injection of rapid-acting insulin. Since the work in [13], that rule has been used in several studies, both clinical and in silico, to tune the gain of a Linear Time Invariant (LTI) model to a particular patient [14-19]. Nevertheless, the 1800 rule is an empirical rule, and the clinical literature does not advise at which glucose concentration it works best, or is most appropriate. This is important because the patient's insulin sensitivity depends, amongst other factors (see [20, 21]), on the glucose concentration, meaning that an LTI representation of the insulin-glucose system is not enough to totally describe it. This nonlinear behavior is illustrated in Fig. 1, where the mean DC gain for all the in silico adults of the UVA/Padova metabolic simulator [22, 23] linearized at several glucose concentrations is depicted. Steady-state glucose concentrations were achieved by only adapting the insulin infusion rate, i.e., the higher the insulin infusion rate, the lower the steady-state glucose concentration, and vice versa. Therefore, the hypoglycemic region presented in Fig. 1 actually represents a hypoglycemic/hyperinsulinemic region, and the hyperglycemic region actually represents a hyperglycemic/hypoinsulinemic region. In order to understand the shape of that figure, both regions can be analyzed separately as follows.

Concerning the hypoglycemic/hyperinsulinemic region, it can be seen from Fig. 1 that there is an increase in insulin sensitivity when glucose decreases

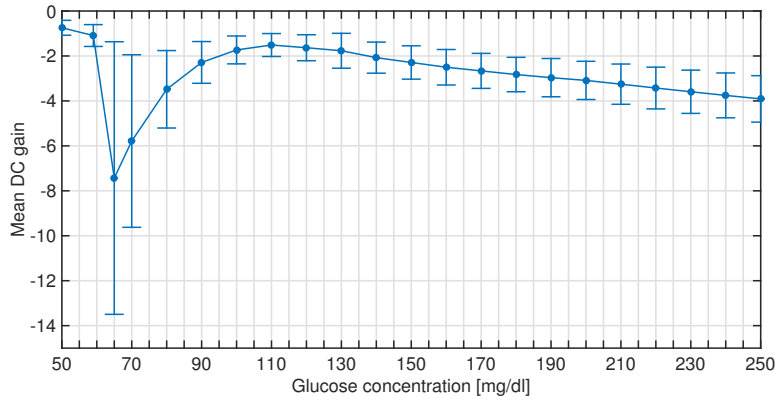

Figure 1: Mean DC gain for the adult patients of the distribution version of the UVA/Padova simulator, linearized at different glucose concentrations. The mean \pm 1 STD values are represented by vertical bars.

below approximately $120 \mathrm{mg} / \mathrm{dl}$. In the UVA/Padova model, it is assumed that the insulin-dependent utilization increases when glucose decreases below its basal value, which is $120 \mathrm{mg} / \mathrm{dl}$ on average. This coincides with clinical knowledge [24, 25]. The loss of insulin sensitivity when glucose decreases below very low concentrations can be explained in the following way. On the one hand, the insulin-dependent utilization in the UVA/Padova model is described considering a "risk" function that increases when glucose decreases below its basal value (the lower the glucose value, the higher the risk), and saturates when glucose reaches $60 \mathrm{mg} / \mathrm{dl}$. On the other hand, there is a counterregulatory response due to the glucagon action. The static secretion of glucagon increases when glucose decreases below its basal value. Together, the increase in glucagon secretion and the saturation of the "risk" function related to the insulin-dependent utilization make the region on the left of Fig. 1 (glucose from 50 to 60 $\mathrm{mg} / \mathrm{dl}$ ) less sensitive to insulin.

Concerning the hyperglycemic/hypoinsulinemic region, we are aware of the basic clinical knowledge that indicates a loss of insulin sensitivity in hyperglycemia. However, it should be considered that such knowledge is generally based on hyperinsulinemic clamps [26, 27], and that a hyperglycemic/hypoinsulinemic event is quite different to many, but not all, real-world hyperglycemic events, which are usually induced by meal intake and are accompanied by prolonged glucose appearance and increased insulin infusion. For example, hyperinsulinemia is associated with insulin receptor deficiency [28, 29], and several works suggest that it is the main inducer of insulin resistance, and not hyperglycemia per se [30-32]. In addition, basing a control law on this case may be safer than basing it on the expectation of reduced insulin sensitivity, because doing so may result in elevated insulin delivery and 
thus may lead to postprandial hypoglycemia.

Multiple Linear Parameter-Varying (LPV) models have been proposed in the past [33-38]. An LPV model is a family of linear time-varying systems described in standard state-space form, with matrices $(A, B, C, D)$ depending on a time-varying parameter vector $\rho(t)$, measured in real time:

$$
\begin{aligned}
& \dot{x}(t)=A(\rho) x(t)+B(\rho) u(t) \\
& y(t)=C(\rho) x(t)+D(\rho) u(t) .
\end{aligned}
$$

LPV models were introduced in the control community in the early 1990s. The first significant results in terms of analysis and controller synthesis can be found in [39-42]. It is a good way to represent a large class of nonlinear models, and particularly, to apply gain-scheduling control in a systematic way, with theoretical guarantees of performance and stability [43]. While in traditional gain-scheduling the gain of a linear controller is adjusted as the operating condition changes (something typically used in aircraft control), in LPV control, a smooth real-time adaptation of the controller to the operating condition is provided. In addition, but at the cost of conservatism, the approach can be applied to an even wider range of systems known as quasi-LPV systems. In this case, the time-varying parameter can be one of the states of the model, in particular the output. Further comments on quasi-LPV models can also be found in [39, 44]. In [33] and [34], the Bergman minimal model [1] was considered and transformed into a quasi-LPV model by an appropriate choice of parameters. In [35-37], the Sorensen compartmental model [2] was linearized at different points, which were defined as the vertexes of an affine-LPV model that covers the original nonlinear one. This model was used as an uncertainty LTI model set, and an $\mathcal{H}_{\infty}$ controller was designed to control it, hence, the time-varying characteristics were not exploited. Finally, in [38], an LPV approach using the Cambridge model [4] was developed.

In this work, the discussion presented in [45] is considered and adapted to the AP application. There, it is explained that the use of complex, high-order models for synthesis is not necessarily related to better closedloop performance. In that sense, a simple third-order LPV model from the insulin delivery input to the glucose deviation output is proposed here, and personalized by a system-oriented reinterpretation of the 1800 rule. Thus, a combination of the model personalization using a priori patient-specific characteristics with the timevarying description of the dynamics by means of an LPV system representation, is achieved. Due to the fact that this modeling strategy is intended mainly for con- troller design, the $\nu$-gap metric $\delta_{\nu}$ (see [46], [47]) is employed to quantify the quality of achievable closed-loop performance afforded by the control-oriented model. Model identification and tuning are performed using the distribution version of the UVA/Padova metabolic simulator. This simulator has been accepted by the U.S. Food and Drug Administration (FDA) as a valid tool usable to test and validate AP control algorithms prior to clinical trials, and has itself been validated in clinical trials [48].

The paper is organized as follows. In Section 2, we summarize the main ideas related to control-oriented models, and describe the procedure to obtain the personalized LPV model. In Section 3, we present open- and closed-loop indexes to quantify the effectiveness of this approach, including comparisons with other controloriented models presented in previous works. A brief discussion and a design example is presented in Section 4, and finally, conclusions are drawn in Section 5.

\section{Materials and methods}

\subsection{Control-oriented models}

The main idea here is based on the discussion presented in [45] and its main reference [49]. There are three fundamental contributions in the last years in the areas of identification and control:

- Seek the best approximate model within a model set, instead of the true system.

- (In)validate the model against the available data.

- Identify based on the particular purpose the model should serve (simulation or control design).

The first point is clearly related to the appearance of robust control during the 1980s, and the second has been instrumental in reducing model uncertainty. The last issue led to the area of control-oriented identification: How to make an a priori selection of the simplest (in some sense, e.g., order, non/linear, time-varying/invariant, deterministic/stochastic) design model in order to achieve the required closed-loop performance. This has been an important research topic for the last 20 years (excellent tutorial in [49]). More recently, robust control-oriented identification deals with the interplay between identification, model uncertainty and worst case performance.

To understand this problem we need to acknowledge the relation between model uncertainty and closed-loop performance for different model structures. The synthesis procedure is applied to the model and produces a controller and a performance index. The idea is then to 
identify the simplest model that approaches the desired performance, rather than a good mimic of the system dynamics.

In addition, coherence between the model and the control design procedure is important. Proposed models with well-established synthesis procedures $\left(\mathcal{H}_{\infty}\right.$, affine and Linear Fractional Transformation (LFT)-LPV control, control commutation) in increasing complexity are:

$$
\text { LTI } \rightarrow \text { affine-LPV } \rightarrow \text { LFT-LPV } \rightarrow \text { switched-LPV }
$$

A first step towards the proposed objective was taken in [50], where model (in)validation is achieved based on the set of controllers that produce a given performance level. There, the initial nominal model was LTI, the performance measure was based on the $\mathcal{H}_{\infty}$-norm, and the set of models was defined in terms of the $\nu$-gap metric, for LTI or Linear Time Varying (LTV) uncertainties. The relation between the size of the model set and the maximum performance level was based on [51].

Extending this result to more general nominal models (LPV), performance requirements and model sets has not been achieved yet. The results in [52] and the identification/invalidation ones in [53] could prove useful. There is still much work to do in producing a performance-oriented identification/invalidation method in order to select the simplest (synthesis) model structure with fewest iterations.

It can be concluded that using complex models for controller synthesis does not necessarily produce better closed-loop performance. A reduced order model simplifies considerably the numerical issues in controller synthesis, particularly when more sophisticated controllers are designed, e.g., time-varying LPV based on Linear Matrix Inequality (LMI) computations.

Control-oriented models used in our work are the ones described in [13], as well as the extensions in [14] and [17]. To quantify the effectiveness of the controller design model, the (unique) metric capable of quantifying the closed-loop performance before the controller is designed has been used: the so-called $\nu$-gap. This measure is briefly described in next subsection.

\section{2. $\nu$-gap metric}

For LTI models, given a controller $K$ and a model $P_{0}$, with $K$ and $P_{0}$ transfer matrices, a performance measure/stability margin for the (stable) closed-loop system $\left(P_{0}, K\right)$ is defined in [46] and [47] as:

$$
b_{P_{0}, K}=\left\|\left[\begin{array}{c}
P_{0} \\
I
\end{array}\right]\left(I-K P_{0}\right)^{-1}\left[\begin{array}{ll}
-K & I
\end{array}\right]\right\|_{\infty}^{-1} \text {. }
$$

where $\|.\|_{\infty}$ indicates the $\mathcal{H}_{\infty}$-norm [54]. A larger $b_{P_{0}, K}$ corresponds to a better performance/stability margin, with the supremum $b_{o p t}=\sup _{K} b_{P_{0}, K}$ its optimal value. An interesting property of the $\nu$-gap (see definition in [46]) is that any controller $K$ stabilizing $P_{0}$ with $b_{P_{0}, K}>\beta$, also stabilizes the model set $\left\{P: \delta_{\nu}\left(P, P_{0}\right) \leq \beta\right\}$. In addition, the difference in the closed-loop performance of a nominal model $P_{0}$ and a perturbed model $P$ for the same controller $K$ can be quantified in terms of $\delta_{\nu}\left(P, P_{0}\right)$, i.e., the smaller $\delta_{\nu}\left(P, P_{0}\right)$, the closer their performances (see details in $[46,47])$. Therefore, this measure quantifies the distance between the closed-loop performance of two different loops without having to design a controller and compare on a one-by-one basis.

In order to quantitatively estimate subjects' insulin sensitivity, a $1 \mathrm{U}$ insulin bolus was applied to each in silico adult of the distribution version of the UVA/Padova simulator at a large number of different steady-state glucose concentrations (operating points), and the maximum unsaturated glucose decrease (glucose drop) was captured in each case. As mentioned before, steady-state glucose concentrations were achieved by only accommodating the insulin infusion rate. For each particular initial glucose concentration, the corresponding initial states of the UVA/Padova model were calculated, and the insulin infusion rate to maintain that glucose level was determined. It is worth remarking that no hypo-treatments, meal or suspension of insulin have been considered under any circumstances. Consequently, the applied method to reach any initial state cannot influence subsequent glucose drops.

The distribution version of the simulator has 11 adults (one, Adult \#11, is an average patient). Because Adult \#007 from the database has an insulin sensitivity that is not coherent with its TDI, it has been excluded, leaving 10 subjects for the following analysis. In Fig. 2, the glucose drop for each patient, and the mean values excluding Adult \#007, are plotted along with the average value of the 1800 rule. The explanation for the shapes presented in this figure is the same as for Fig. 1. Note that Adult \#007 is the patient most sensitive to insulin, despite having a TDI of $43 \mathrm{U}$, which is close to the mean TDI dose of around $46 \mathrm{U}$. It is worth mentioning that the Correction Factor $(\mathrm{CF})$ implemented in the simulator follows the 1800 rule (1800/TDI), even though it is stated (seemingly erroneously) in [23] that the simulator's CF is characterized according to $1700 / \mathrm{TDI}$.

\subsection{Average model}

As shown in Fig. 2, the 1800 rule is only rendered correct at $235 \mathrm{mg} / \mathrm{dl}$. Hence, for each adult of the 


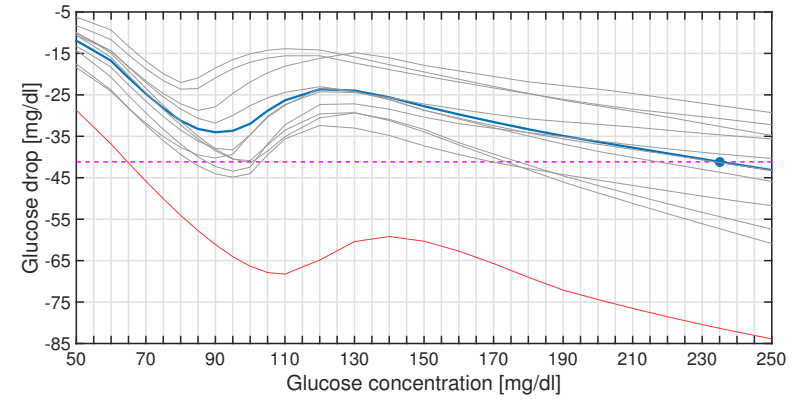

Figure 2: Glucose drop for each in silico adult (gray lines: Study patients, red line: Adult \#007) and the mean values excluding Adult \#007 (blue line) at different operating points after a $1 \mathrm{U}$ insulin bolus. The magenta dashed line indicates the average value resulting from the 1800 rule.

UVA/Padova simulator, a linearized model from the subcutaneous-insulin delivery ( $\mathrm{pmol} / \mathrm{min}$ ) to the subcutaneous-glucose concentration deviation $(\mathrm{mg} / \mathrm{dl})$ is calculated at this operating point. Subsequently one LTI model, based on the mean of the frequency responses of each subject's frequency response, is identified. In order to obtain a simple low-order system, a grey-box identification method was performed using the Matlab System Identification Toolbox ${ }^{\mathrm{TM}}$ with the following model structure:

$$
G(s)=k \frac{s+z}{\left(s+p_{1}\right)\left(s+p_{2}\right)\left(s+p_{3}\right)} \mathrm{e}^{-15 s} .
$$

The identified parameters are $k=-1.6788 \times 10^{-5}$, $z=0.1501, p_{1}=0.0035, p_{2}=0.0138$, and $p_{3}=$ 0.0143 , achieving a $98.58 \%$ fitting. The goodness of the fit expressed as a percentage has been obtained by:

$\mathrm{FIT}=100\left(1-\frac{\left\|y_{p}-y\right\|}{\|y-\bar{y}\|}\right)$

where $y$ is the measured output data, $\bar{y}$ is its mean, $y_{p}$ is the predicted output, and $\|$.$\| indicates the 2-norm. Note$ that the structure of model (3) is similar to the structure of previous control-oriented models [13, 14, 17] that have led to successful control as verified in clinical trials. The objective of this paper is to improve on the control performance via a superior model, but without unnecessarily increasing the model order. In Fig. 3, the Nyquist plots of both the average frequency response and the identified model are depicted.

The bandwidth (BW) of a system is commonly defined as the first frequency satisfying $-3 \mathrm{~dB}$ from DC gain. Here, we use that definition to represent the insulin sensitivity variation detected in Figs. 1 and 2, by making the BW of the proposed model (3) vary

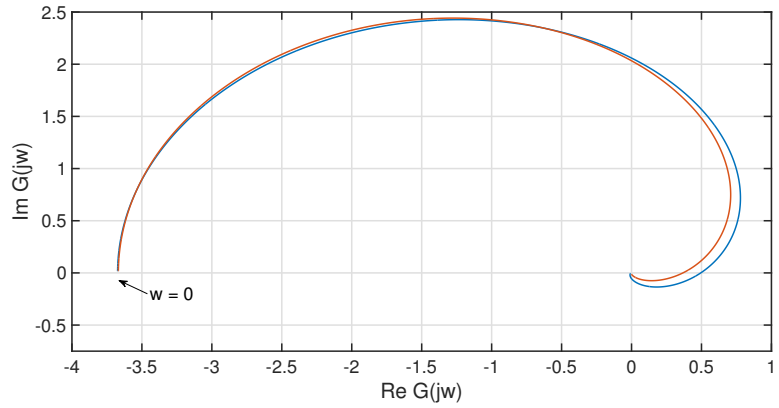

Figure 3: Nyquist plots of the mean frequency response of the linearized models (light-blue) and the identified LTI model (orange).

with subcutaneous-glucose concentration $g[\mathrm{mg} / \mathrm{dl}]$ appropriately. In this way, the average BW (denoted $\overline{\mathrm{BW}}$ ) variation of the linearized models at different glucose values was obtained, and fitted with a goodness of fit of $91.23 \%$ accuracy by the following continuous, piecewise polynomial function:

$\overline{\mathrm{BW}}(g)=a_{i} g^{3}+b_{i} g^{2}+c_{i} g+d_{i}$

where:

$i= \begin{cases}1 & \text { if } 300 \mathrm{mg} / \mathrm{dl} \leq g \\ 2 & \text { if } 110 \mathrm{mg} / \mathrm{dl} \leq g<300 \mathrm{mg} / \mathrm{dl} \\ 3 & \text { if } 65 \mathrm{mg} / \mathrm{dl} \leq g<110 \mathrm{mg} / \mathrm{dl} \\ 4 & \text { if } 59 \mathrm{mg} / \mathrm{dl} \leq g<65 \mathrm{mg} / \mathrm{dl} \\ 5 & \text { if } g<59 \mathrm{mg} / \mathrm{dl} .\end{cases}$

In this way, data is adjusted with good accuracy without the use of a high-order function. Results are illustrated in Fig. 4, and parameter values are given in Table 1. It should be remarked that here, the goodness of the fit has been assessed considering a vector of glucose values from 40 to $400 \mathrm{mg} / \mathrm{dl}$ (inclusive) in steps of $1 \mathrm{mg} / \mathrm{dl}$.

According to Fig. 4, the average BW has a similar shape to the average DC gain depicted in Fig. 1. For example, in the region where the absolute value of the gain is larger (between 65 and $80 \mathrm{mg} / \mathrm{dl}$ ), the $\mathrm{BW}$ is lower. Observe that, as shown in Figs. 1 and 4, there is an abrupt change at $60 \mathrm{mg} / \mathrm{dl}$. This behavior was explained in the Introduction.

For fixed parameters, the DC gain of model (3) is $\frac{k z}{p_{1} p_{2} p_{3}}$. We introduce a simple manner of replicating the glucose dependent bandwidth, by allowing one parameter, $p_{1}$, to be selected and thereby reproduce the measured values. A decrease in the model's BW is associated with a decrease in the value of $p_{1}$, and as a consequence, an increase in the absolute value of the model's static gain. 
Table 1: Parameter values of $\overline{\mathrm{BW}}(g)$ of (5).

\begin{tabular}{ccccc}
\hline$i$ & $a_{i}$ & $b_{i}$ & $c_{i}$ & $d_{i}$ \\
\hline 1 & 0 & 0 & $-2.6291 \times 10^{-6}$ & $3.8947 \times 10^{-3}$ \\
2 & 0 & $4.5505 \times 10^{-8}$ & $-2.8536 \times 10^{-5}$ & $7.5712 \times 10^{-3}$ \\
3 & $-2.8294 \times 10^{-8}$ & $7.3020 \times 10^{-6}$ & $-5.6072 \times 10^{-4}$ & $1.5967 \times 10^{-2}$ \\
4 & 0 & 0 & $-7.0925 \times 10^{-4}$ & $4.8702 \times 10^{-2}$ \\
5 & 0 & 0 & $-5.7447 \times 10^{-6}$ & $7.1954 \times 10^{-3}$ \\
\hline
\end{tabular}

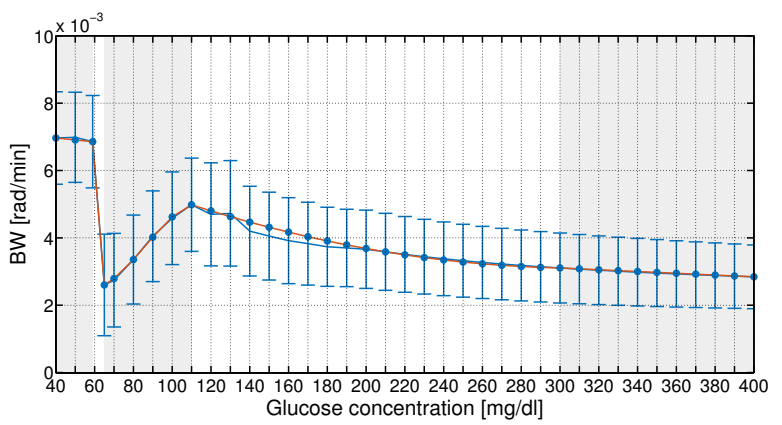

Figure 4: Piecewise polynomial function of the average bandwidth $\overline{\mathrm{BW}}(g)$ (orange line), and average variation of the $\mathrm{BW}$ for the in silico adults linearized at different glucose values (light-blue line). Vertical lines represent the average $\mathrm{BW} \pm 1 \mathrm{STD}$.

The next step is to characterize the dependence of parameter $p_{1}$ on glucose $g$ in order to make the BW of model (3) coincide with the piecewise function $\overline{\mathrm{BW}}(g)$ for any given value of $g$. To this end, for a glucose concentration $g^{*}$, a desired $\mathrm{BW} w^{*}$ is defined as $\overline{\mathrm{BW}}\left(g^{*}\right)$. Then, parameter $p_{1}$ is computed so that:

$$
\frac{\left|\frac{j w^{*}}{z}+1\right|}{\left|\frac{j w^{*}}{p_{1}}+1\right|\left|\frac{j w^{*}}{p_{2}}+1\right|\left|\frac{j w^{*}}{p_{3}}+1\right|}=10^{-3 / 20} .
$$

Note that Eqn. (7) is normalized by its DC gain, and that $10^{-3 / 20}$ is equivalent to $-3 \mathrm{~dB}$ expressed in magnitude units. Parameter $p_{1}$ was calculated by repeating this procedure for multiple values of $g$, and then fitting with a $98.00 \%$ accuracy by the following function:

$p_{1}(g)=q_{i} g^{3}+r_{i} g^{2}+s_{i} g+t_{i}$

with $i$ defined as in Eqn. (6). Here, once again, a vector of glucose from 40 to $400 \mathrm{mg} / \mathrm{dl}$ in steps of $1 \mathrm{mg} / \mathrm{dl}$ has been used for assessing the goodness of the fit. Results are illustrated in Fig. 5, and parameter values are given in Table 2. It is worth remarking that it is not the change in the bandwidth that affects the DC gain, but the change in the parameter used to define the bandwidth $\left(p_{1}\right)$.
Table 2: Parameter values of $p_{1}(g)$ of (8)

\begin{tabular}{ccccc}
\hline$i$ & $q_{i}$ & $r_{i}$ & $s_{i}$ & $t_{i}$ \\
\hline 1 & 0 & 0 & $-3.4321 \times 10^{-6}$ & $4.4706 \times 10^{-3}$ \\
2 & 0 & $9.0580 \times 10^{-8}$ & $-5.3562 \times 10^{-5}$ & $1.1357 \times 10^{-2}$ \\
3 & $-4.2382 \times 10^{-8}$ & $1.1402 \times 10^{-5}$ & $-9.1676 \times 10^{-4}$ & $2.5849 \times 10^{-2}$ \\
4 & 0 & $1.7321 \times 10^{-4}$ & $-2.3080 \times 10^{-2}$ & $7.7121 \times 10^{-1}$ \\
5 & 0 & 0 & $-2.8336 \times 10^{-5}$ & $1.4083 \times 10^{-2}$ \\
\hline
\end{tabular}

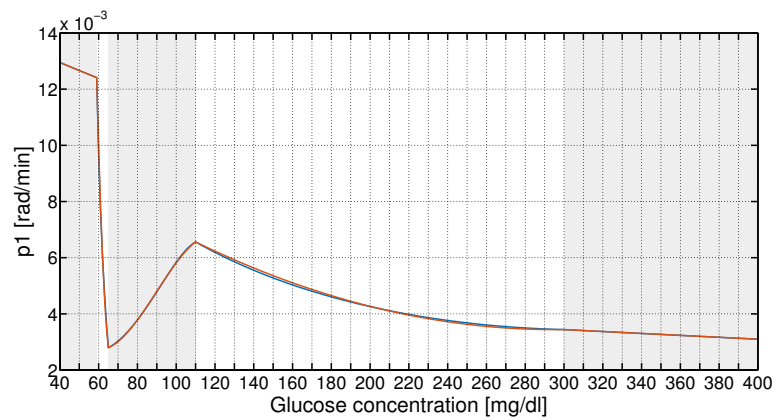

Figure 5: Parameter $p_{1}$ computed at different values of glucose concentration $g$ (light-blue), and piecewise polynomial function $p_{1}(g)$ (orange).

Finally, an average LPV model with the following state-space representation can be obtained by including the glucose-varying parameter $p_{1}(g)$ into the model structure (3):

$$
\begin{aligned}
\dot{x}(t) & =A\left(p_{1}\right) x(t)+B u_{\Delta}(t) \\
y_{\Delta}(t) & =C x(t)
\end{aligned}
$$

with

$$
\begin{aligned}
A\left(p_{1}\right)= & {\left[\begin{array}{ccc}
0 & 1 & 0 \\
0 & 0 & 1 \\
0 & -p_{2} p_{3} & -\left(p_{2}+p_{3}\right)
\end{array}\right] } \\
& +p_{1}\left[\begin{array}{ccc}
0 & 0 & 0 \\
0 & 0 & 0 \\
-p_{2} p_{3} & -\left(p_{2}+p_{3}\right) & -1
\end{array}\right] \\
B= & {\left[\begin{array}{lll}
0 & 0 & 1
\end{array}\right]^{T}, \quad C=k\left[\begin{array}{lll}
z & 1 & 0
\end{array}\right], }
\end{aligned}
$$

and with $u_{\Delta}(t)=u(t)-u_{\mathrm{op}}$, and $y_{\Delta}(t)=y(t)-y_{\mathrm{op}}$ being, respectively, the difference between the insulin delivery input $u(t)$ and the glucose deviation output $y(t)$ from the operating point $\left\{u_{\mathrm{op}}, y_{\mathrm{op}}\right\}$. Note that model (9) is affine in the parameter $p_{1}(g)$, and that a delay of 15 min should be added to the output (see (3)). In addition, it is worth mentioning that if $p_{1}(g)$ is evaluated at $g=235 \mathrm{mg} / \mathrm{dl}$, it produces a result $(0.0038)$ that is slightly different from the value previously obtained with the grey-box identification method (0.0035). Thus, 


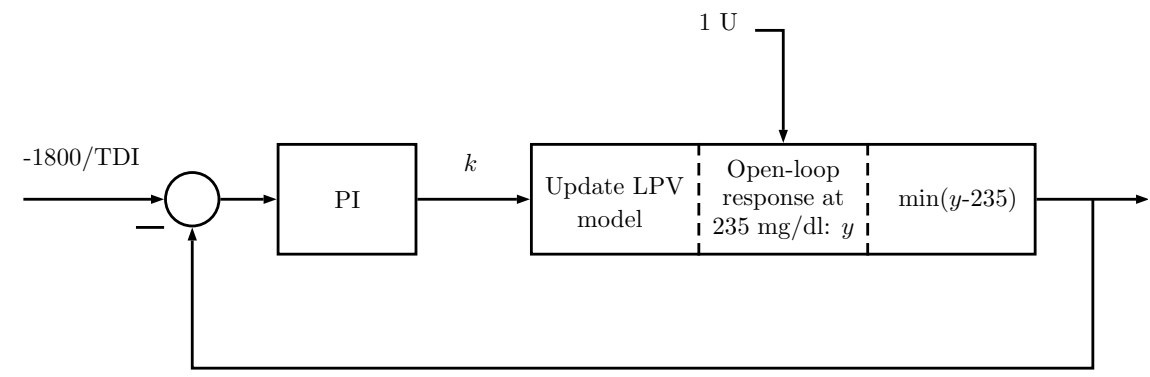

Figure 6: Control loop to update gain $k$. It is composed of two blocks: the left-one that represents the PI controller, and right-one that represents the methodology followed to capture the glucose drop. Concerning the latter, first, the LPV model is updated with the current value of $k$, then the time-response to a $1 \mathrm{U}$ insulin bolus is obtained, and finally, its minimum value (the glucose drop) is captured.
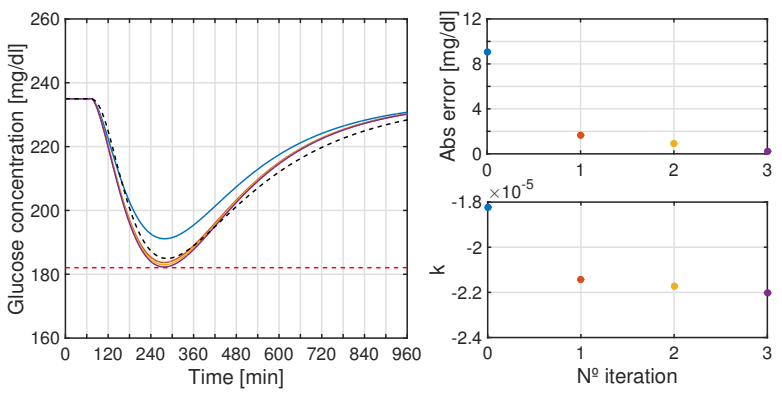

Figure 7: Tuning procedure of parameter $k$ for Adult \#009. Left: Average model (light-blue line); nonlinear model (black dashed line); set-point based on the 1800 rule (red dashed line). Right-top: Absolute error between desired and actual glucose. Right-bottom: Evolution of parameter $k$.

in order to maintain coherence with the static gain of the LTI model previously identified, the static gain of the LTI model resulting from holding parameter $p_{1}$ fixed at 0.0038 is consistently adjusted by modifying the value of parameter $k$ from $-1.6788 \times 10^{-5}$ to $-1.8244 \times 10^{-5}$.

\subsection{Model tuning}

As mentioned before, the interpatient variability should be considered in modeling the insulin-glucose dynamics, and in consequence, the model should be tuned to each patient. To this end, the following procedure is carried out. For each in silico Adult $\# j$, its TDI is selected from the UVA/Padova simulator database, and defined as $\operatorname{TDI}_{j}$. Then, the 1800 rule, i.e., $1800 / \mathrm{TDI}_{j}$, indicates the maximum glucose drop to be reached by the personalized LPV model when it is excited with a $1 \mathrm{U}$ insulin bolus at a steady-state glucose concentration $g$ of $235 \mathrm{mg} / \mathrm{dl}$. Finally, the tuning method consists in computing a suitable gain $k$ that makes the model achieve that condition.
An intuitive and simple way to approach this problem is by means of a control loop, like the one depicted in Fig. 6, where a discrete Proportional-Integral (PI) controller with transfer-function $3.5 \times 10^{-7}\left(1+\frac{1}{z-1}\right)$ is used to adjust the value of $k$. An analytical procedure could also be used for this same purpose, but has not been explored here. In summary, Fig. 6 represents a basic closed-loop diagram, where:

- the reference is the glucose drop due to a 1 $\mathrm{U}$ insulin bolus indicated by the 1800 rule (1800/TDI ${ }_{j}$ );

- the output is the glucose drop obtained with the LPV model after a $1 \mathrm{U}$ insulin bolus; and

- the gain $k$ is the control signal.

The gain $k$ is not continuously updated. First, the LPV model is defined with the current value of $k$, which is initialized with its average value $k=-1.8244 \times 10^{-5}$. Then, the open-loop response to a $1 \mathrm{U}$ insulin bolus is obtained, the operating point $g=235 \mathrm{mg} / \mathrm{dl}$ is subtracted, and thereafter, the minimum value (the glucose drop) is captured. The latter sequence is represented by the larger block of the diagram. Finally, the reference is compared with the obtained glucose drop, and if there is a relative error greater than the predefined threshold of $5 \times 10^{-3}$, a new value of $k$ is calculated by means of the PI controller, closing the loop. As an example, the process to obtain $k$ for Adult \#009 is illustrated in Fig. 7. Finally, the personalized values of gain $k$ for all in silico adults are presented in Table 3.

\section{Results}

\subsection{Open-loop comparison}

For each of the 10 virtual adult patients of the distribution version of the UVA/Padova simulator, an 
Table 3: Personalized gain $k$ for each in silico adult.

\begin{tabular}{rrrr}
\hline Adult \#j & $k \times 10^{5}$ & Adult $\# j$ & $k \times 10^{5}$ \\
\hline 001 & -1.7888 & 006 & -1.0343 \\
002 & -1.7451 & 008 & -1.4379 \\
003 & -1.4343 & 009 & -2.2024 \\
004 & -2.1396 & 010 & -1.5919 \\
005 & -1.8650 & 011 & -1.8864 \\
\hline
\end{tabular}

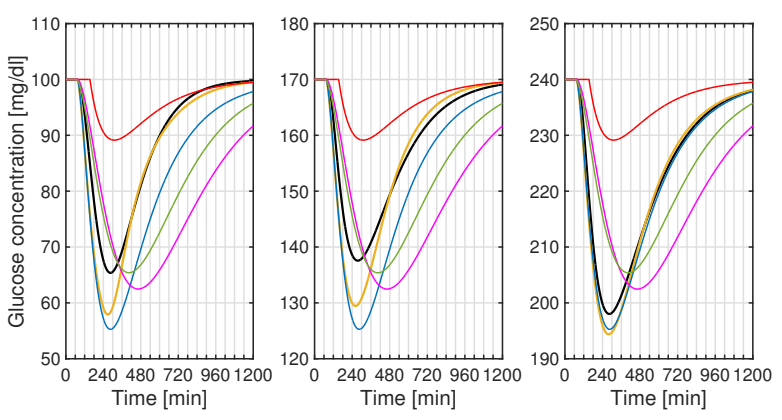

Figure 8: Response to a $1 \mathrm{U}$ insulin bolus starting from 100, 170 and $240 \mathrm{mg} / \mathrm{dl}$ for Adult \#011, considering models (i) (orange), (ii) (lightblue), (iii) (green), (iv) (red), (v) (magenta), and the UVA/Padova nonlinear model (black line).

insulin bolus of $B \mathrm{U}$, with $B \in\{0.5,1,1.5\}$, was applied at different operating points to test (i) the personalized LPV model. A comparison with, (ii) the average LTI model in (3), and (iii) the model presented in [13], as well as (iv) and (v), the extensions described in [14] and [17], respectively, are considered. As an example, in Fig. 8, the response to a $1 \mathrm{U}$ insulin bolus of all those models starting at 100, 170 and $240 \mathrm{mg} / \mathrm{dl}$ for Adult \#011 (average patient) are depicted. It can be observed that with $240 \mathrm{mg} / \mathrm{dl}$ as an operating point, the average LTI and the proposed personalized LPV have similar behavior. Nevertheless, the BW adjustment in the LPV model provides a much better fit to the original nonlinear model, when the concentration moves away from that condition.

For each patient at each operating point, the RMSE between the time-response of each control oriented model $\left(y_{p}\right)$ and the nonlinear UVA/Padova model $(y)$ to an insulin bolus of $B \mathrm{U}$ is calculated as:

$\mathrm{RMSE}=\frac{\left\|y_{p}-y\right\|}{\sqrt{n}}$

where $\|$.$\| indicates the 2-norm, and n$ is the number of samples. Here, in order to capture the complete glucose variation from the operating point, $n$ has been defined as 2880 , considering a sampling time of $1 \mathrm{~min}$. Then,
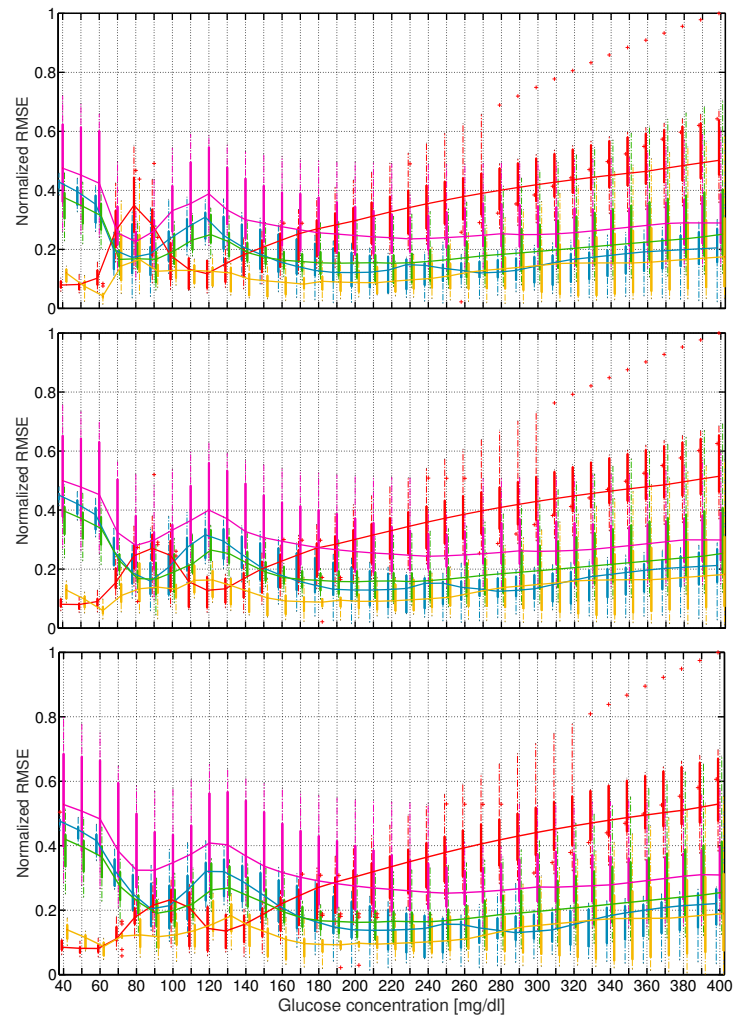

Figure 9: Normalized RMSE between the time-response of the control-oriented models (i) (orange), (ii) (light-blue), (iii) (green), (iv) (red), and (v) (magenta), and the nonlinear UVA/Padova description to an insulin bolus of amplitude $0.5 \mathrm{U}$ (above), $1 \mathrm{U}$ (middle), and 1.5 $\mathrm{U}$ (below) at different operating points. The continuous lines indicate the medians, and the red crosses the outliers. The vertical bars are limited by the 25 th and 75 th percentiles.

the normalized RMSE (NRMSE) is computed as:

$\mathrm{NRMSE}=\frac{\mathrm{RMSE}}{\mathrm{RMSE}_{\max }}$

with $\mathrm{RMSE}_{\max }$ being the maximum RMSE obtained for each value of $B$. In Fig. 9, the median value of the normalized RMSE and its dispersion for all 10 patients at different operating points are illustrated. It is worth noting that the LPV model has the best fit for most of the glucose concentration values that were considered. The model in [14] also presents good performance in regions where, according to Fig. 2, $g$ does not tend to drop significantly. This is because that model is less sensitive to insulin than the others, and, as a consequence, is compensated by an Insulin Feedback Loop (IFL) when controlled. Instead, the average LTI model reaches its best performance at large values of $g$, due to the fact that it was identified at a high glucose level. 
Table 4: Model comparison in terms of the $\delta_{\nu}$. Improvement is over model (3), the average LTI model.

\begin{tabular}{|c|c|c|c|c|c|}
\hline & \multirow{2}{*}{ Model } & \multicolumn{2}{|c|}{$[70-180] \mathrm{mg} / \mathrm{dl}$} & \multicolumn{2}{|c|}{$[40-400] \mathrm{mg} / \mathrm{dl}$} \\
\hline & & Average $\delta_{\nu}$ & Improvement (\%) & Average $\delta_{\nu}$ & Improvement (\%) \\
\hline (i) & Proposed Personalized LPV model & 0.1681 & 44.21 & 0.1599 & 30.10 \\
\hline (vi) & Personalized LTI model & 0.2566 & 14.84 & 0.2363 & 3.75 \\
\hline (ii) & Average LTI model in (3) & 0.3013 & 0.00 & 0.2455 & 0.00 \\
\hline (iii) & Model presented in [13] & 0.3044 & -1.03 & 0.3357 & -36.74 \\
\hline (v) & Model described in [17] & 0.4057 & -34.65 & 0.4167 & -69.74 \\
\hline (iv) & Model described in [14] & 0.4144 & -37.54 & 0.5434 & -121.34 \\
\hline
\end{tabular}

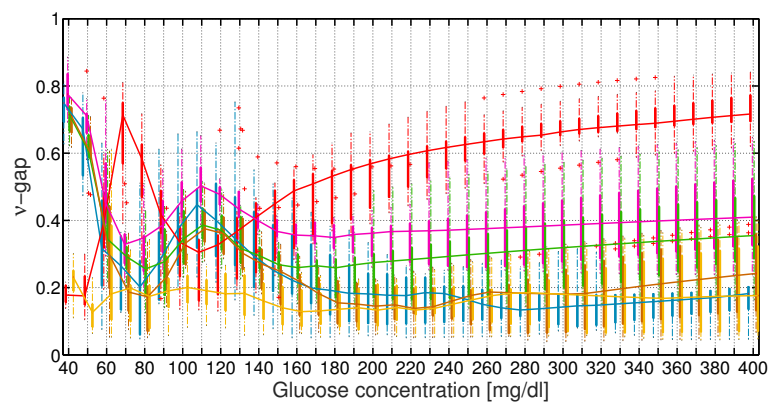

Figure 10: $\nu$-gap $\delta_{\nu}$ between the UVA/Padova model linearized at different glucose concentrations and models (i) (orange), (ii) (lightblue), (iii) (green), (iv) (red), (v) (magenta), and (vi) (brown). The continuous lines indicate the medians, and the red crosses the outliers. The vertical bars are limited by the 25 th and 75 th percentiles.

\subsection{Closed-loop comparison}

The comparison of different model representations of a physical system for simulation purposes is well established, and has been explored in the previous subsection. Nevertheless, when the model is intended for controller design, the comparison is not so obvious. A good model, i.e., one that fits adequately a set of experimental data (in this case a $\mathrm{Hi}-\mathrm{Fi}$ simulator as the UVA/Padova one), does not necessarily provide a good model for control design [45]. For this reason, we have used the $\nu$-gap metric $\delta_{\nu}[46,47]$ that considers the distance between two models according to their achievable closed-loop performance (see Section 2.2).

Here, due to the fact that the UVA/Padova simulator has been linearized at different glucose concentrations $g$, the $\delta_{\nu}$ distance between each linearized model and the personalized LPV description has been computed as a function of $g$. For comparison purposes, the distance between the simulator and models (i)-(vi), where (vi) is the average LTI model (3) personalized by its gain $k$ using the 1800 rule, is also calculated. This is illustrated in Fig. 10, where the median value of the $\nu$-gap and its dispersion for all 10 patients is depicted in each case. Note that this figure indicates an important improvement in (i), (ii) and (vi) with respect to the other three models. In addition, among models (i), (ii) and (vi), the improvement goes from (ii) $\rightarrow$ (vi) $\rightarrow$ (i), being therefore, the tuned LPV model, the one with the lowest $\delta_{\nu}$. The main differences between them lie in the glucose region $[90,180] \mathrm{mg} / \mathrm{dl}$ and at $50 \mathrm{mg} / \mathrm{dl}$. The average values, and the relative improvement with respect to model (ii), are indicated in Table 4 for the complete glucose range of $[40,400] \mathrm{mg} / \mathrm{dl}$ and for a normal glucose target range of $[70,180] \mathrm{mg} / \mathrm{dl}$.

Finally, note that although model (iv) presents less RMSE than the other LTI models in several situations according to Fig. 9, this does not imply that it is better for designing glucose controllers, as reflected in Table 4.

\section{Discussion}

The LPV model presented in this work has been considered in the design of a switched Linear Quadratic Gaussian (LQG) controller that has been successfully tested on the complete in silico adult cohort of the UVA/Padova metabolic simulator, and in the clinical trial announced in [55].

Here, closed-loop simulations are presented, considering models (i), (iii), (iv) and (v) at the design stage. However, note that a comparison of model (i) and any other model cannot lead to a definitive proof of improvement in closed-loop performance. In a way, it is similar to the comparison of a robust controller with a nominal one, e.g., $\mathcal{H}_{\infty}$ vs. $\mathcal{H}_{2}$ optimal controllers. The performance strongly depends on which model has been selected to represent the physical system. If the worst case model from the uncertainty set is selected and tested, the nominal controller can be proven closed-loop unstable while the robust control could provide a good performance. Instead, if the nominal model used to design the $\mathcal{H}_{2}$ optimal control is tested, then its performance can be arbitrarily better than the robust controller, depending of the size of the uncertainty set. Hence, a fair comparison should be in terms of how the assumption on the system representation has been expanded, e.g., from a nominal model to a set of models. Therefore, several comments are in order to place the example into an appropriate context. 
- The proposed personalized LPV model reflects the time-varying and nonlinear nature of the problem, which is not the case with the previous controloriented models.

- The previous fact allows to take advantage of this more accurate description of the problem, therefore provides the possibility of designing, with an adequate synthesis procedure, a controller that exploits this time-varying characteristic, and hence, yield better performance. Of course, if the possibility is not exploited the result could be worst.

- The result of the comparison always depends on many factors, e.g., the design weights selected, as in the case of robust vs. nominal controller tests as described before.

In our case then, the assumption on the underlying dynamics has been expanded from LTI to LTV, in particular LPV.

In this example, the Matlab Robust Control Toolbox $^{\mathrm{TM}}$ was used to compute the controllers. A switched LPV controller that switches between two LPV controllers is synthesized considering model (i), and a switched $\mathcal{H}_{\infty}$ controller that switches between two $\mathcal{H}_{\infty}$ controllers is obtained for each model (iii)-(v). Both control strategies are composed of two controllers: one conservative (controller 1) that performs slight changes on the patient's basal insulin infusion rate, and one aggressive (controller 2) that is selected at the time of meal ingestion to generate an insulin spike, and in consequence, reduce postprandial hyperglycemia risks.

The switched LPV controller is designed following the procedure presented in Section II.B in [17], but in this case, with model (i) as the patient design model, and with the following LTI performance and actuator weights:

$$
\begin{aligned}
W_{p}(s) & =100 \frac{10 s+1}{1000 s+1} \\
W_{u, k}(s) & =\frac{\alpha_{k}}{0.01 s+1}
\end{aligned}
$$

where $\alpha_{k}=\{1,0.035\}$. Thus, the actuator weight $W_{u, 1}$ related to controller 1 is defined more conservatively than the actuator weight $W_{u, 2}$ related to controller 2. The parameter $p_{1}$ is constrained to lie with the interval $[0.0028,0.013]$ based on the expected minimum and maximum glucose values $(40-400 \mathrm{mg} / \mathrm{dl})^{1}$.

${ }^{1}$ Actual blood glucose may go lower than $40 \mathrm{mg} / \mathrm{dl}$ and higher than
The same performance and actuator weights are used for the $\mathcal{H}_{\infty}$ controller design, but, in each case, the augmented model is defined considering models (iii), (iv), and (v), respectively. Because a continuous-time synthesis procedure is followed, discrete-time models (iii) and (iv) are converted to continuous-time models at the design stage. However, in all cases, the control system operates in discrete-time with a sample-period $T_{s}=5 \mathrm{~min}$, therefore the derived continuous-time control law is converted to a discrete-time control law later at implementation.

As mentioned before, controller 2 is applied only at meal times to generate an insulin spike akin to the standard open-loop basal-bolus treatment. The problem at this point is how a meal-related situation is detected. Here, a simple approach is followed where the meal time is announced. This signal triggers the switched controller from controller 1 to controller 2, which will command the insulin infusion during $30 \mathrm{~min}$. After that controller 1 will automatically take over the insulin delivery.

Closed-loop simulations start at midnight with the patient's basal glucose concentration at a constant setpoint, and a meal of $50 \mathrm{~g}$ of carbohydrates is ingested at $0700 \mathrm{~h}$. In addition, CGM and a Continuous Subcutaneous Insulin Infusion (CSII) pump are used as sensor and actuator, respectively. The additive, stochastic CGM noise is created with the same random seed in all simulations for comparison purposes, and the carry-over scheme described in [18] is applied to the insulin command to accommodate the pump discretization of $0.1 \mathrm{U}$.

The closed-loop responses for all in silico adults of the distribution version of the UVA/Padova simulator are presented in Fig. 11 and the Control Variability Grid Analysis (CVGA) plots [56] are illustrated in Fig. 12. In order to analyze the glucose variability, the Blood Glucose Risk Index (BGRI) is calculated for each case [57]. It is found that while for the closed-loop responses obtained considering model (i), the BGRI is 1.3 on average, for models (iii), (iv), and (v), the BGRIs are 2.6, 12.8, and 2.4, respectively, on average. Thus, in this case, the best performance in terms of glucose variability and risk of hypoglycemia is obtained with the switched LPV controller. However, as discussed at the beginning of this section, the main contribution here is a controller design model that includes time-varying characteristics of the original problem and that has a good match with the system. This allows the possibility

$400 \mathrm{mg} / \mathrm{dl}$, however, current Continuous Glucose Monitoring (CGM) devices return a measurement only with the interval $[40,400] \mathrm{mg} / \mathrm{dl}$. 


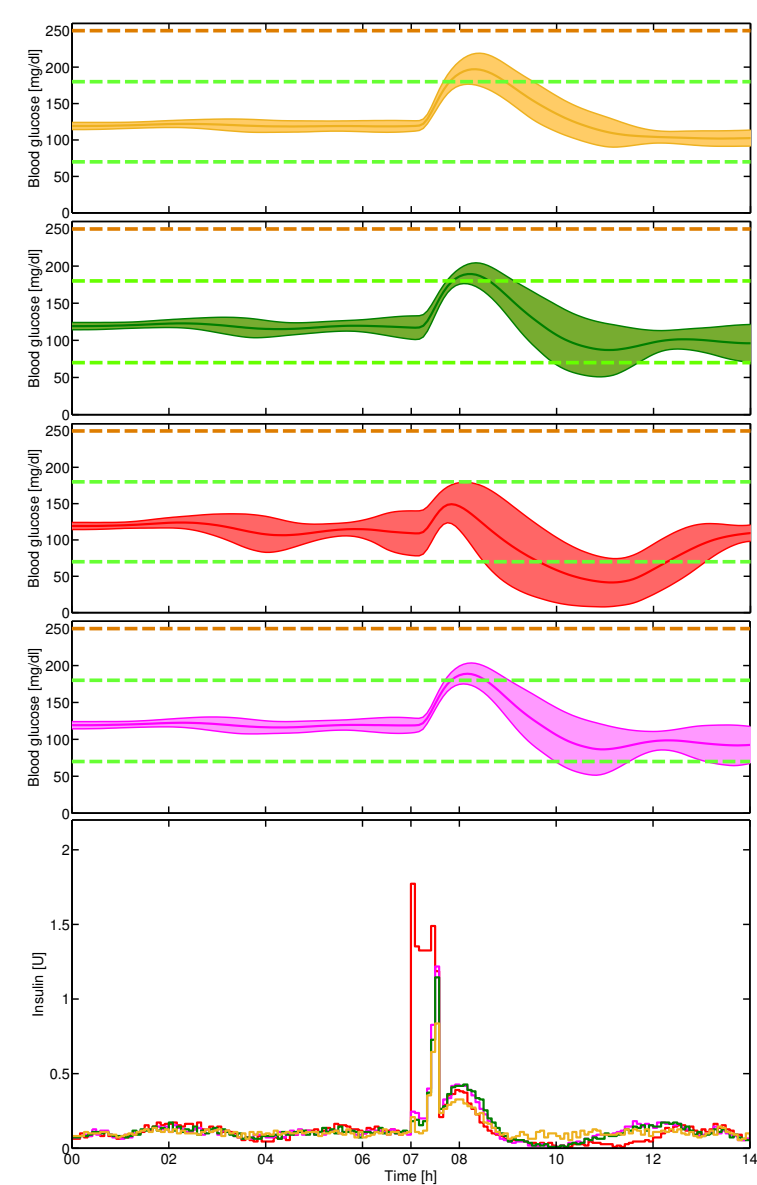

Figure 11: Average closed-loop responses for all in silico adults of the distribution version of the UVA/Padova model to a meal of $50 \mathrm{~g}$ at $0700 \mathrm{~h}$, considering models (i) (orange), (iii) (green), (iv) (red), and (v) (magenta). Above: The thick lines are the mean blood glucose values, and the boundaries of the filled areas are the mean \pm 1 STD values. Dashed lines (green and orange) represent the limits of the 70$180 \mathrm{mg} / \mathrm{dl}$ and 70-250 mg/dl ranges. Below: Average insulin infusion.

of having a better performance, if an adequate design procedure is used.

\section{Conclusions}

A control-oriented LPV model, based on the UVA/Padova metabolic model, that is affine in the parameter $p_{1}$ was proposed. This parameter is itself a more general function of the glucose level, which can be measured in real-time. This anticipates a better means of designing LPV controllers to achieve a higher closed-loop performance in the T1DM control problem. The main benefit of employing the proposed LPV model is that the time-varying characteristics of the problem dynamics can be considered at the control law

\begin{tabular}{lrrrrr}
\hline & A-zone & B-zone & C-zone & D-zone & E-zone \\
\hline Stars & $27.3 \%$ & $72.7 \%$ & $0 \%$ & $0 \%$ & $0 \%$ \\
Circles & $27.3 \%$ & $54.5 \%$ & $9.1 \%$ & $9.1 \%$ & $0 \%$ \\
Squares & $9.1 \%$ & $0 \%$ & $81.8 \%$ & $9.1 \%$ & $0 \%$ \\
Diamonds & $18.2 \%$ & $45.5 \%$ & $9.1 \%$ & $27.3 \%$ & $0 \%$ \\
\hline
\end{tabular}

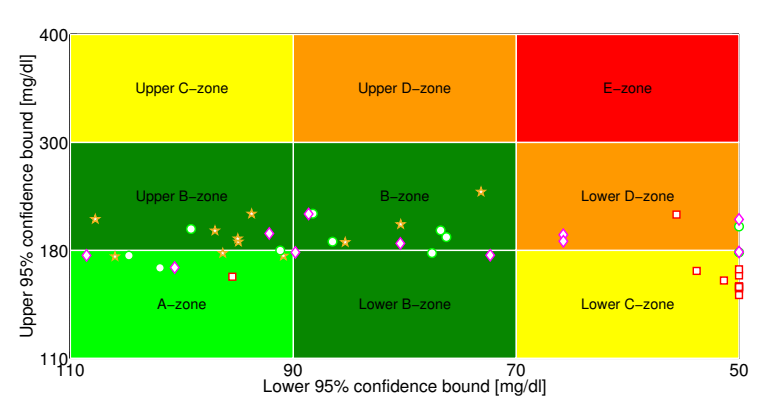

Figure 12: CVGA plots of the closed-loop responses for all in silico adults of the distribution version of the UVA/Padova model to a meal of $50 \mathrm{~g}$ at $0700 \mathrm{~h}$, considering models (i) (stars), (iii) (circles), (iv) (squares), and (v) (diamonds).

synthesis stage. Therefore, the use of this model is expected to induce a better closed-loop performance if an appropriate control design procedure is applied.

The proposed, personalized LPV model was compared to previous control-oriented models in an openloop fashion, by measuring the RMSE with the UVA/Padova distribution version simulator. Also a closed-loop comparison quantified by the $\nu$-gap was made. In both cases, the proposed personalized LPV model achieved smaller errors, possibly due to the fact that time-varying dynamics and a novel generalization and re-interpretation of the well-known 1800 rule were considered.

Finally, it is worth remarking that although the UVA/Padova simulator has some limitations because of the high-complex and uncertain dynamics associated with T1DM, it has been accepted by the U.S. FDA as a valid tool usable to test control algorithms for artificial pancreas, and has itself been validated in clinical trials.

\section{Conflict of interest}

No competing financial interests exist.

\section{Acknowledgement}

P. Colmegna and R. S. Sánchez-Peña were supported by CONICET, and by Nuria (Argentina) and Cellex (Spain) Foundations. 


\section{References}

[1] R. N. Bergman, Y. Ider, C. Bowden, C. Cobelli, Quantitative estimation of insulin sensitivity, Am. J. Physiol. 236 (6) (1979) E667-677.

[2] J. T. Sorensen, A physiologic model of glucose metabolism in man and its use to design and assess improved insulin therapies for diabetes, Ph.D. thesis, Massachusetts Institute of Technology, Cambridge, MA, USA (1985).

[3] C. Dalla Man, R. Rizza, C. Cobelli, Meal simulation model of the glucose-insulin system, IEEE Trans. Biomed. Eng. 54 (10) (2007) 1740-1749.

[4] R. Hovorka, F. Shojaee-Moradie, P. Carroll, L. Chassin, I. Gowrie, N. Jackson, R. Tudor, A. Umpleby, R. Jones, Partitioning glucose distribution/transport, disposal, and endogenous production during IVGTT, Am. J. Physiol. Endocrinol. Metab. 282 (5) (2002) E992-1007.

[5] C. Cobelli, E. Renard, B. P. Kovatchev, Artificial pancreas: Past, present, future, Diabetes 60 (11) (2011) 2672-2682.

[6] S. D. Patek, B. W. Bequette, M. Breton, B. A. Buckingham, E. Dassau, F. J. Doyle III, J. Lum, L. Magni, H. C. Zisser, In silico preclinical trials: Methodology and engineering guide to closed-loop control in type 1 diabetes mellitus, J. Diabetes. Sci. Technol. 3 (2) (2009) 269-282.

[7] P. G. Fabietti, V. Canonico, M. O. Federici, M. M. Benedetti, E. Sarti, Control oriented model of insulin and glucose dynamics in type 1 diabetics, Med. Biol. Eng. Comput. 44 (1-2) (2006) 69-78.

[8] E. Dassau, H. Zisser, R. Harvey, M. Percival, B. Grosman, W. Bevier, E. Atlas, S. Miller, R. Nimri, L. Jovanovič, F. J. Doyle III, Clinical evaluation of a personalized artificial pancreas, Diabetes Care 36 (4) (2013) 801-809.

[9] S. Schaller, S. Willmann, J. Lippert, L. Schaupp, T. R. Pieber, A. Schuppert, T. Eissing, A generic integrated physiologically based whole-body model of the glucose-insulin-glucagon regulatory system, CPT: Pharmacometrics \& Syst. Pharmacol. 2 (8) (2013) 1-10. doi:10.1038/psp.2013.40.

[10] S. S. Kanderian, S. A. Weinzimer, G. M. Steil, The identifiable virtual patient model: Comparison of simulation and clinical closed-loop study results, J. Diabetes Sci. Technol. 6 (2) (2012) 371-379.

[11] Q. Wang, P. Molenaar, S. Harsh, K. Freeman, J. Xie, C. Gold, M. Rovine, J. Ulbrecht, Personalized state-space modeling of glucose dynamics for type 1 diabetes using continuously monitored glucose, insulin dose, and meal intake: An extended Kalman filter approach, J. Diabetes Sci. Technol. 8 (2) (2014) 331-345

[12] J. Walsh, R. Roberts, Pumping Insulin, 4th Edition, Torrey Pines Press, San Diego, CA, 2006.

[13] K. van Heusden, E. Dassau, H. C. Zisser, D. E. Seborg, F. J. Doyle III, Control-relevant models for glucose control using $a$ priori patient characteristics, IEEE Trans. Biomed. Eng. 59 (7) (2012) 1839-1849.

[14] J. Lee, E. Dassau, D. Seborg, F. J. Doyle III, Model-based personalization scheme of an artificial pancreas for type 1 diabetes applications, in: American Control Conference (ACC), Washington, DC, USA, 2013, pp. 2911-2916.

[15] R. Gondhalekar, E. Dassau, H. C. Zisser, F. J. Doyle III, Periodic-zone model predictive control for diurnal closed-loop operation of an artificial pancreas, J. Diabetes Sci. Technol. 7 (6) (2013) 1446-1460.

[16] P. Colmegna, R. S. Sánchez-Peña, R. Gondhalekar, E. Dassau, F. J. Doyle III, Reducing risks in type 1 diabetes using $\mathcal{H}_{\infty}$ control, IEEE Trans. Biomed. Eng. 61 (12) (2014) 2939-2947.

[17] P. Colmegna, R. S. Sánchez-Peña, R. Gondhalekar, E. Dassau,
F. J. Doyle III, Switched LPV glucose control in type 1 diabetes, IEEE Trans. Biomed. Eng. 63 (6) (2016) 1192-1200.

[18] R. Gondhalekar, E. Dassau, F. J. Doyle III, Periodic zone-MPC with asymmetric costs for outpatient-ready safety of an artificial pancreas to treat type 1 diabetes, Automatica 52 (9) (2016) 237246.

[19] P. Colmegna, R. S. Sánchez-Peña, R. Gondhalekar, E. Dassau, F. J. Doyle III, Reducing glucose variability due to meals and postprandial exercise in T1DM using switched LPV control: In silico studies, J. Diabetes Sci. Technol. 10 (3) (2016) 744-753.

[20] Y. C. Kudva, R. E. Carter, C. Cobelli, R. Basu, A. Basu, Closedloop artificial pancreas systems: Physiological input to enhance next-generation devices, Diabetes Care 37 (5) (2014) 11841190.

[21] F. J. Doyle III, L. M. Huyett, J. B. Lee, H. C. Zisser, E. Dassau, Closed-loop artificial pancreas systems: Engineering the algorithms, Diabetes Care 37 (5) (2014) 1191-1197.

[22] B. P. Kovatchev, M. Breton, C. Dalla Man, C. Cobelli, In silico preclinical trials: A proof of concept in closed-loop control of type 1 diabetes, J. Diabetes Sci. Technol. 3 (1) (2009) 44-55.

[23] C. Dalla Man, F. Micheletto, D. Lv, M. Breton, B. Kovatchev, C. Cobelli, The UVA/PADOVA type 1 diabetes simulator: New features, J. Diabetes Sci. Technol. 8 (1) (2014) 26-34.

[24] B. Kovatchev, L. Farhy, D. Cox, M. Straume, V. Yankov, L. Gonder-Frederick, W. Clarke, Modeling insulin-glucose dynamics during insulin induced hypoglycemia. evaluation of glucose counterregulation, J. Theor. Med. 1 (4) (1999) 313-323.

[25] B. Kovatchev, M. Straume, L. Farhy, D. Cox, Dynamic network model of glucose counterregulation in subjects with insulinrequiring diabetes, Methods Enzymol. 321 (2000) 396-410.

[26] E. Donga, O. Dekkers, E. Corssmit, J. Romijn, Insulin resistance in patients with type 1 diabetes assessed by glucose clamp studies: systematic review and meta-analysis, Eur. J. Endocrinol. 173 (1) (2015) 101-119.

[27] I. Schauer, J. Snell-Bergeon, B. Bergman, D. Maahs, A. Kretowski, R. Eckel, M. Rewers, Insulin resistance, defective insulin-mediated fatty acid suppression, and coronary artery calcification in subjects with and without type 1 diabetes: The CACTI study, Diabetes 60 (1) (2011) 306-314.

[28] J. Gavin, J. Roth, D. N. Jr., P. de Meyts, D. Buell, Insulindependent regulation of insulin receptor concentrations: a direct demonstration in cell culture, Proc. Natl. Acad. Sci. U.S.A. 71 (1) (1974) 84-88

[29] A. Soli, C. Kahn, D. N. Jr., J. Roth, Insulin receptor deficiency in genetic and acquired obesity, J. Clin. Invest. 56 (4) (1975) 769-780.

[30] A. Davidoff, M. Davidson, M. Carmody, M. Davis, J. Ren, Diabetic cardiomyocyte dysfunction and myocyte insulin resistance: role of glucose-induced PKC activity, Mol. Cell Biochem. 262 (1-2) (2004) 155-163.

[31] Y. Lee, J. Fluckey, S. Chakraborty, M. Muthuchamy, Hyperglycemia- and hyperinsulinemia-induced insulin resistance causes alterations in cellular bioenergetics and activation of inflammatory signaling in lymphatic muscle, FASEB J. 31 (7) (2017) 2744-2759.

[32] H. Liu, S. Cao, T. Hong, J. Han, Z. Liu, W. Cao, Insulin is a stronger inducer of insulin resistance than hyperglycemia in mice with type 1 diabetes mellitus (T1DM), J. Biol. Chem. 284 (40) (2009) 27090-27100.

[33] L. Kovács, B. Kulcsár, J. Bokor, Z. Benyó, LPV fault detection of glucose-insulin system, in: 14th Mediterranean Conference on Control and Automation, Ancone, Italy, 2006, pp. 1-5.

[34] R. S. Sánchez-Peña, A. Ghersin, LPV control of glucose for diabetes type I, in: IEEE EMBS (Ed.), 32nd Annual International Conference, Buenos Aires, Argentina, 2010, pp. 680-683.

[35] L. Kovács, B. Kulcsár, LPV modeling of type I diabetes melli- 
tus, in: 8th International Symposium of Hungarian Researchers, Budapest, Hungary, 2007, pp. 163-173.

[36] L. Kovács, B. Kulcsár, J. Bokor, Z. Benyó, Model-based nonlinear optimal blood glucose control of type I diabetes patients, in 30th Annual International IEEE EMBS Conference, Vancouver, BC, Canada, 2008, pp. 1607-1610.

[37] L. Kovács, B. Benyó, J. Bokor, Z. Benyó, Induced $\mathcal{L}_{2}$-norm minimization of glucose-insulin system for type I diabetic patients, Comput. Meth. Progr. Bio. 102 (2) (2011) 105-118.

[38] P. Szalay, G. Eigner, L. A. Kovács, Linear matrix inequalitybased robust controller design for type-1 diabetes model, in: 19th IFAC World Congress, Cape Town, South Africa, 2014, pp. 9247-9252.

[39] J. Shamma, M. Athans, Gain scheduling: Potential hazards and possible remedies, IEEE Control Syst. Mag. 12 (3) (1992) 101107.

[40] P. Gahinet, P. Apkarian, An LMI approach to $\mathcal{H}_{\infty}$ control, Int. J. Robust Nonlin. 4 (8) (1994) 421-448.

[41] G. Becker, A. Packard, Robust performance of LPV systems using parametrically-dependent linear feedback, Syst. Control Lett. 23 (1994) 205-215.

[42] A. Packard, Gain scheduling via linear fractional transformations, Syst. Control Lett. 22 (2) (1994) 79-92.

[43] S. Shahruz, S. Behtash, Design of controllers for linear parameter varying systems by the gain scheduling technique, J. Math. Anal. Appl. 168 (1) (1992) 195-217.

[44] R. Toth, H. Seddik, H. Werner, On the state-space realization of LPV input-output models: Practical approaches, IEEE Trans. Autom. Control 20 (1) (2012) 139-153.

[45] R. S. Sánchez-Peña, F. Bianchi, Model selection: from LTI to switched-LPV, in: American Control Conference (ACC), Montreal, Canada, 2012, pp. 1561-1566.

[46] G. Vinnicombe, Frequency domain uncertainty and the graph topology, IEEE Trans. Autom. Control 38 (9) (1993) 13711383.
[47] G. Vinnicombe, Uncertainty and Feedback: $\mathcal{H}_{\infty}$ Loop-shaping and the $\nu$-gap metric, Imperial College Press, London, 2001.

[48] C. Dalla Man, F. Micheletto, D. Lv, M. Breton, B. Kovatchev, C. Cobelli, The university of Virginia/Padova type 1 diabetes simulator matches the glucose traces of a clinical trial, Diabetes Technol. Ther. 16 (7) (2014) 428-434.

[49] M. Gevers, Identification for control: From the early achievements to the revival of experiment design, Eur. J. Control 11 (2005) 1-18.

[50] J. Steele, G. Vinnicombe, Closed-loop time-domain model validation in the nu-gap metric, in: Proceedings of the Conference on Decision and Control, 2001, pp. 4332-4337.

[51] K. Glover, D. McFarlane, Robust stabilization of normalized coprime factor plant descriptions with $\mathcal{H}_{\infty}$-bounded uncertainty, IEEE Trans. Autom. Control 34 (8) (1989) 821-830.

[52] S. D. Douma, P. Van den Hof, Relations between uncertainty structures in identification for robust control, Automatica 41 (2005) 439-457.

[53] F. Bianchi, R. S. Sánchez Peña, Robust identification/invalidation in an LPV framework, International J. of Robust and Nonlinear Control. 20 (3) (2010) 301-312.

[54] R. S. Sánchez-Peña, M. Sznaier, Robust systems theory and applications, Wiley, New Jersey, 1998

[55] R. Sánchez-Peña, P. Colmegna, L. Grosembacher, M. Breton, H. De Battista, F. Garelli, W. Belloso, E. Campos-Náñez, V. Simonovich, V. Beruto, P. Scibona, D. Cherñavvsky, Artificial Pancreas: First clinical trials in Argentina, in: 20th IFAC World Congress, Toulouse, France, 2017, pp. 7997-8002.

[56] L. Magni, D. M. Raimondo, C. Dalla Man, M. Breton, S. Patek, G. De Nicolao, C. Cobelli, B. P. Kovatchev, Evaluating the efficacy of closed-loop glucose regulation via control-variability grid analysis, J. Diabetes Sci. Technol. 2 (4) (2008) 630-635.

[57] W. Clarke, B. Kovatchev, Statistical tools to analyze continuous glucose monitor data, Diabetes Technol. Ther. 11 (Suppl. 1) (2009) S45-S54. 